\title{
PRRS in Vietnam and Its Diagnosis
}

\author{
Ngoc Hai Nguyen ${ }^{1}$, Thi Hong Vi Vuong ${ }^{2}$ and Tan Hung $\mathrm{Vo}^{2}$ \\ 1. Department of Infectious Diseases and Public Health, Faculty of Animal Science and Veterinary Medicine, Nong Lam University, \\ Ho Chi Minh city 0084-8, Vietnam \\ 2. HanViet Veterinary Diagnosis Laboratory, Nong Lam University, Ho Chi Minh city 0084-8, Vietnam
}

Received: August 19, 2014 / Accepted: June 16, 2015 / Published: June 30, 2015.

\begin{abstract}
Genotyping 42 serum samples taken from the pigs in the oubreaks from 2009 to 2013 with RT-PCR and nested RT-PCR, showed more than $80 \%$ of samples were positive with Chinese PRRSV clade, and the others were European and North American classical PRRSV genotypes. Ten serum samples from unnapprent PRRS herds were examined for antibodies against PRRSV with ELISA and also for PRRSV with RT-PCR. It was clearly that the titer of antibodies against PRRSV by ELISA test could not be used for interpreting PRRSV infection. In despite of PRRS vaccination or non-vaccination, a risk of PRRSV infection and re-infection exist, utilizing RT-PCR in combination with serology will give the producer and veterinarian PRRSV more exact situation in the herds.
\end{abstract}

Key words: Vietnam, PRRS, genotype, diagnosis, ELISA, RT-PCR.

\section{Introduction}

PRRS (porcine reproductive and respiratory syndrome) caused by a small, enveloped RNA virus belonging to the Arteriviridae family, order Nidovirales, has become a serious challenge to the global pig industry, causing serious economic losses. In Vietnam, PRRS virus has been being perhaps on pigs imported from the U.S. in 1997. Since then up to 2013, PRRS has spread quickly and influenced very seriously the livestock industry in Vietnam. From the surveillance data, that revealed a "new" PRRSV Chinese variant in northern of Vietnam, whilst in the southern part of the country a mix of new Chinese and older classical NA PRRSV variants was detected [1]. Because of the important economical losses by PRRS and the widely spread of disease, a massive vaccination with MLV vaccines is applied in most of the pig sectors that could cause some problems of virus persistence in the herds. And furthermore, the vaccination and biosecurity practices are not as good

Corresponding author: Ngoc Hai Nguyen, Ph.D., assistant professor, research field: swine diseases. E-mail: nguyenngochai@hcmuaf.edu.vn. as in all pig industrial farms and smallholders, that present opportunities for PRRSV spread in whole country. The efficacy of PRRS vaccine is very often discussed by the problem of homogenous or heterogenous PRRSV strains and genotypes. The complexity of PRRS control with vaccination in the pig industry causes not only because of the variety of PRRSV strains and genotypes infection in different pig herds, but also because of combination of PRRSV genotypes infection in the pig herds. Actually, both attenuated live and inactivated vaccines are commercially available, but it is important to match the genotype of the vaccine with that circulating in the pig population [1]. However, vaccination of pigs does not prevent PRRSV infection, it may reduce clinical disease and transmission of the wild-type virus. It is important to note that the modified-live vaccine virus can persist in pigs and be disseminated through semen and oral fluids; it should therefore not be used in naïve herds, pregnant sows or breeding gilts and boars [1]. The study is realized to know how the PRRSV spreads in the country by the times and the risk of virus persistence in the herds applied MLV vaccines. The 
results would be considered in taking the measures of PRRS control.

\section{Materials and Methods}

The serum samples were taken from pigs presenting with or without clinical symptomes (42 samples in the cases of PRRS outbreak, and 10 samples in the unapparent cases of PRRS) in the herds that either was PRRS vaccinated or non-vaccinated.

Primers used in nested RT-PCR to differenciate NA (North American) and EU (European) genotypes were with outer primers [2] of F1: ATGGCCAGCC AGTCAATC and R1: TCGCCCTAATTG AT AGGTG, and inner primers [3] were F2: AGTCC AGAG GCAAGGGACCG and R2: TCAATCAG TGCCATT CACCAC, and F3: ATGATAAAG TCCCAGCGC CAG and R3: CTGTATGAGCAAC CGGCAGCAT. And to determine Chinese clade the using designed primers [4] were F: CGACGAGCTT AAAGACCAGATGG and R: CATCACAAGCCT CACGCATGA. The PCR products size expected for the primers F1 and R1 (PRRSV, NA and EU), F2 and R2 (PRRSV, NA), F3 and R3 (PRRSV, EU), and F and $\mathrm{R}$ for Chinese clade were $433 \mathrm{bp}, 337 \mathrm{bp}$, and 241 bp, 757 bp, respectively.

RT-PCR: Basic components of the RT-PCR reaction of $\mathrm{MgCl}_{2}(25 \mathrm{~mm}) 0.7 \mu \mathrm{L}$; dNTP's $(10 \mathrm{~mm})$ each $0.7 \mu \mathrm{L}$; primer $\mathrm{F}(20 \mu \mathrm{m})$, primer $\mathrm{R}(20 \mu \mathrm{m})$, each $0.4 \mu \mathrm{L}$. The reaction was made in two phases:
Phase 1 , running the RT to obtain cDNA with the following parameters $50{ }^{\circ} \mathrm{C} / 30 \mathrm{~min} ; 95{ }^{\circ} \mathrm{C} / 15 \mathrm{~min}$, phase 2 running PCR with 35 cycles: $95{ }^{\circ} \mathrm{C} / 10$ seconds; $55{ }^{\circ} \mathrm{C}$ of 30 seconds; $72{ }^{\circ} \mathrm{C} / 20$ seconds and then run at $72{ }^{\circ} \mathrm{C} / 3$ min and $25{ }^{\circ} \mathrm{C} / 30$ seconds. Keep products in $4{ }^{\circ} \mathrm{C}$. Electrophoresis of products realized on $1.5 \%$ agarose gel at $85 \mathrm{~V}$ for $35 \mathrm{~min}$ (Gel Doc, Bio-rad). Results observed compared with ladder (Promega) $100 \mathrm{bp}$ in size and the positive control. In the case if routine RT-PCR was negative, an one time PCR addition was realized to amplify more ADN product. ELISA kit (IDEXX) was used for determine the antibody titer in serum.

\section{Results}

\subsection{Genotypes of PRRSV}

Total 42 serum samples taken from the pigs in the oubreaks from 2009 to 2013 for PRRSV genotyping with nested RT-PCR to differentiate the European and North American PRRS genotype (Fig. 1), and with RT-PCR to determine either the North American classical or Chinese PRRSV clade (Fig. 2). The results of RT-PCR and nested RT-PCR showed that most of the PRRS cases cause by the PRRSV strains which belong to Chinese clade. The results showed more than $80 \%$ the positive cases was Chinese PRRSV clade, and there was two cases that was positive with both European and North American PRRS genotype (Table 1).

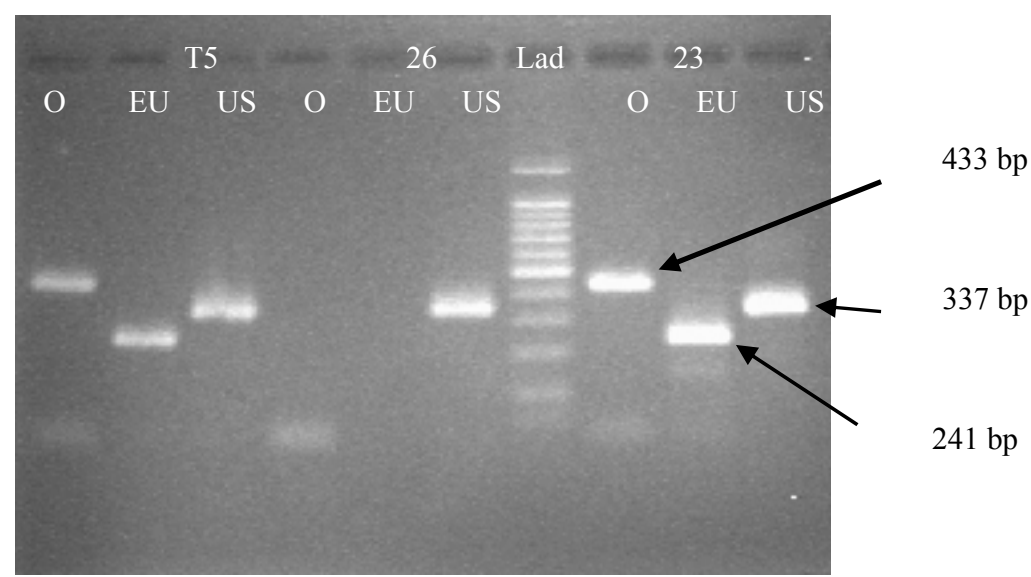

Fig. 1 Nested RT-PCR for PRRSV genotyping. Lad: ladder; O: with outer primers; EU: with European PRRSV primers; US: with North American PRRSV primers. 


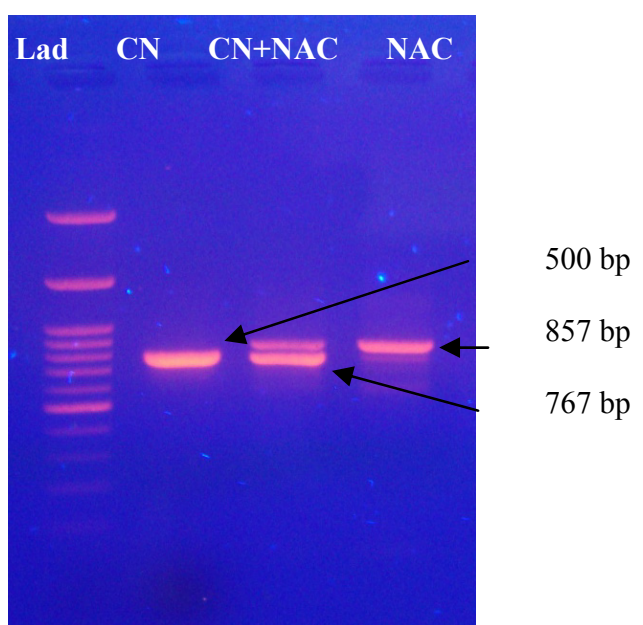

Fig. 2 RT-PCR for Chinese PRRSV genotyping. CN: Chinese clade; NAC: North American classical clade.

\subsection{ELISA and RT-PCR PRRS Diagnosis}

Ten serum samples from unnapprent PRRS herds were examined for antibodies against PRRSV with ELISA and also for PRRSV with RT-PCR and to know whether the antibodies titer with ELISA could interpret the PRRS situation in the herds. By the results showed in Table 2, it was clearly that the titer of antibodies against PRRSV by ELISA test does not present the capacity of PRRSV protection in the pig herds. All of ten samples was negative with routine RT-PCR for PRRSV, but was positive for PRRSV with modified RT-PCR (RT-PCR with one time PCR addition) (Table 2). RT-PCR is a very sensitive method in PRRSV diagnosis but there is always a limited sensitivity of every method. In a unapparent pig herds, the viral circulation is so low in blood that the virus could be detected with conventional method. A suitable diagnostic method is very important to determine the PRRSV exact situation in the herds.

\section{Discussion and Conclusions}

The most severity of the PRRS in Vietnam was observed in the second episode from 2007 to 2010. At that times the vaccination against to PRRS was not applied as principal measure to prevent this disease, in particular for the sows. But, from 2010, most of the

Table 1 Genotypes of PRRSV.

\begin{tabular}{lllll}
\hline Source & Total & EU & \multicolumn{2}{c}{ NA } \\
\cline { 4 - 5 } & & & Classical clade & Chinese clade \\
HCM city, 2009 & 19 & 0 & 4 & 15 \\
HCM city, 2010 & 12 & $2^{*}$ & $4^{*}$ & 8 \\
Bac giang, 2010 & 4 & 0 & 0 & 4 \\
Ha noi, 2010 & 2 & 0 & 0 & 5 \\
Long an, 2013 & 5 & 0 & 0 & 5 \\
\hline
\end{tabular}

$(*)$ : two samples was positive with both European and North American PRRS genotype.

Table 2 PRRS diagnosis with ELISA, RT-PCR methods.

\begin{tabular}{llll}
\hline Post-weaning & ELISA,$+/-,(\mathrm{S} / \mathrm{P})$ & RT-PCR & RT-PCR* \\
\hline 1 & $-(0.164)$ & - & + \\
2 & $-(0.108)$ & - & + \\
3 & $-(0.024)$ & - & + \\
4 & $+(0.43)$ & - & + \\
5 & $-(0.013)$ & - & + \\
\hline Growing-finisher & & & + \\
\hline 1 & $+(1.295)$ & - & + \\
2 & $+(1.901)$ & - & + \\
3 & $+(1.858)$ & - & + \\
4 & $+(2.201)$ & - & + \\
5 & $+(2.147)$ & - & + \\
\hline
\end{tabular}

- (RT-PCR*): RT-PCR with one time PCR addition. 
pig farms, the industrial as well as the small ones, has applied the vaccination against PRRS for the sows, with the results that the oubreaks and also the severity caused by PRRS were not important as in episode 2007-2010. However, the PRRS vaccination was realized periodically only in the industrial sector, so that the PRRS oubreaks have been occured still up to now in the pig farms of small-scale where there are the poor biosecurity practices. So the role of small-scale holdings for virus persistence, in particular, in high pig density areas will require special attention. That could be the reason for continuous PRRS outbreaks in Vietnam, and the culling has been shown to be a less effective measure, and massive PRRS vaccination of susceptible animals should be considered as more suitable measure to control PRRS in Vietnam. The same picture was founded by FAO in their report in 2011 [1].

The PRRSV, before the PRRS outbreak in China, 2006, are classified in EU and NA genotypes, in which all the strains belong to the NA genotypes are known just only for one clade. But after appearance of PRRSV Chinese strains, because of the mutation of these PRRSV strains with a deletion of about one hundred of nucleotides on ORF1a, the new PRRSV Chinese strains, although they are always belong to PRRSV NA genotype, they are divided into PRRSV Chinese clade to differentiate with PRRSV NA classical. In 2006/07, PRRSV Chinese clade caused a very important PRRS epidemic in China and later in Vietnam. And the spread pattern in Vietnam (2007-2010) suggests the occurrence of new strains in the northern part of the country earlier in March 2007 [1]. The presence of PRRSV Chinese clade was also demonstrated in the study of Nguyen Ngoc Hai and Vo Khanh Hung, 2012. There is a multitype of PRRS genotype infection in the studied pig herds: (i) infection with one genotype of North American Chinese PRRSV (65.22\%); (ii) infection with one genotype of North American PRRSV (32.60\%); (iii) infection with two genotypes of North American and
European PRRSVs (1.28\%) [5].

The results of the present study presented the complexity of PRRSV infection genotype in the Vietnamese pig herds. A multitype of PRRS genotype infection in the pig herds makes the difficulties not only in diagnosis but also in vaccine application to control the PRRS. At present, there are different types of PRRS vaccine that contain the modified live virus vaccine of EU, NA classical, and NA Chinese strains, but there are no clear evidence for which PRRS vaccine that could give a best protection for PRRS in Vietnam. However, the diagnosis that could determine the PRRSV genotype can help the pig farmers in choosing a most suitable vaccination strategy for their pig herds.

Normally, in Vietnam, after vaccination the farmers are interested in antibodies titer to predict the PRRS situation in their pig herds. If ELISA results are positive with high $\mathrm{S} / \mathrm{P}$ values, the farmers will believe in that their pig herds should be protective from PRRS and there is no PRRSV circulation in their pig herds. In fact, this is not true. By the results shown in Table 3, the PRRSV antibodies titer determined by ELISA was not a good indicator for PRRS protection. By K-J Yoon et al. [6], none of the available serologic tests can differentiate positive results due to infection from positive results due to vaccination. The PRRS vaccination could not totally protect the vaccinated herds from PRRSV persistence or re-infection. A modified-live virus vaccine PRRS has the potential shedding of vaccine virus to other susceptible populations of swine in direct or indirect contact with vaccinated swine. Martina Velasova et al. [7], had found the PRRSV positive in serum samples taken from the PRRS vaccinated farms as weel as from the non-vaccinated farms. And the farms using PRRSV live vaccine had higher odds of being PRRSV positive compared to nonvaccinated farms. In general, until active virus infection, there is lacking of clinical signs and poor recognition of clinical signs of PRRS in the PRRS 
vaccinated pig herds that could contribute to PRRSV infection remaining undetected on farms. As the result, instituting PRRS-specific control measures could be delayed on such farms and they may, therefore, remain a source of infection to other pig units and also have a risk of PRRS outbreak.

The results shown in the present study demontrated that antibodies serotesting could not present exactly the PRRS situation in the pig herds. There is no correlation between ELISA antibodies against PRRSV and virus existence. The antibodies seronegative does not mean absence of virus infection in the herds, and the high titer of antibodies does not mean the herds could be protected or free of virus infection. The PRRS vaccination could decrease clinical problem in pigs, but it could cause the persistence and circulation of virus vaccine, or even PRRSV field strains in the herds. Detecting the virus in any case of infection is very important to institute PRRS effective control measures.

\section{Conclusions}

In despite of PRRS vaccination or non-vaccination, when herd monitoring is desired, a combination of nested RT-PCR and serology testing will be more valuable in determination of virus infection herd status.

\section{Acknowledgements}

Gratefully thanks to the HanViet Veterinary
Diagnosis Laboratory, Nong Lam University for technical support.

\section{References}

[1] Dietze, K., Pinto J., Wainwright, S., and Hamilton, C. 2011. "Porcine Reproductive and Respiratory Syndrome (PRRS): Virulence Jumps and Persistent Circulation in Southeast Asia. In Focus on... Rome." Food and Agriculture Organization of the United Nations 5: 8.

[2] Mardassi, H., Mounir, S., and Dea, S. 1994. "Identification of Major Differences in the Nucleocapsid Protein Genes of a Quebec Strain and European Strains of Porcine Reproductive and Respiratory Syndrome Virus." J. Gen. Virol. 75: 681-5.

[3] Truyen, U., Wilhelm, S., Genzow, M., and Schagemann, G. 2006. "Porcine Reproductive and Respiratory Syndrome Virus (PRRSV): A Ring Test Performed in Germany to Assess RT-PCR Detection Methods.” J. Vet. Med. B. 53: 68-74.

[4] Feng, Y., Zhao, T., Nguyen, T., Inui, K., Ma, Y., Nguyen, T. H., Nguyen, V. C., Liu, D., Bui, Q. A., To, L. T., Wang, C., Tian, K., and Gao, G. F. 2008. "Porcine Respiratory and Reproductive Syndrome Virus Variants, Vietnam and China, 2007." Emer. Infect. Diseases 14 (11): 1774-6.

[5] Nguyen, N. H., and Vo, K. H. 2012. "Genetic Variety of PRRS Virus Infection in Some Pig Herds." Veterinary Sciences and Techniques 1: 20-6.

[6] Yoon, K. J., Christopher-Hennings, J., and Nelson, E. A. 2003. Diagnosis of PRRS Virus. 2003 PRRS Compendium Producer Edition.

[7] Velasova, M., Alarcon, P., Williamson, S., and Wieland, B. 2012. "Risk Factors for Porcine Reproductive and Respiratory Syndrome Virus Infection and Resulting Challenges for Effective Disease Surveillance." $B M C$ Veterinary Research 8: 184. 